\title{
Alternative Treatment Approach for Right Heart Masses
}

\author{
Abhishek Bhagat ${ }^{1}$, Frank H. Annie ${ }^{2}$, Alfred Tager ${ }^{3}$, Aravinda Nanjundappa ${ }^{4}$, Christopher Adams ${ }^{2}$ \\ 1. Internal Medicine, Charleston Area Medical Center, Charleston, USA 2. Cardiology, Charleston Area Medical Center, \\ Charleston, USA 3. Emergency Medicine, Charleston Area Medical Center, Charleston, USA 4. Cardiology, Charleston \\ Area Medical Center / West Virginia University, Charleston, USA
}

Corresponding author: Frank H. Annie, frank.h.annie@camc.org

\begin{abstract}
The traditional therapeutic approach for heart masses has been surgical resection. For right-sided masses, percutaneous mechanical thrombectomy (PMT) is a viable treatment option which is being applied with increasing frequency. This newer treatment modality is less invasive, less expensive, and results in shorter hospital stays compared to cardiac surgery. We demonstrate below a case in which rheolytic PMT was utilized successfully, allowing the patient to be discharged the following day.
\end{abstract}

Categories: Cardiology

Keywords: pmt, heart mass

\section{Introduction}

Heart masses (abnormal growth in or adjacent to the heart) as well as opiate epidemic are increasing in frequency in aging population. These heart masses are classified into three categories: Tumor, thrombus and vegetation. Tumors are of malignant or benign etiology [1]. Thrombi can be of type A which are highly mobile and associated with deep venous thrombosis (DVT)/pulmonary embolism (PE) or type B which tend to be non-mobile and form in situ [1-4]. The third variety of heart masses is vegetation which is primarily caused by bacterial infections. These lesions are frequently identified upon workup for endocarditis, stroke, murmur, or are incidental findings. For most heart masses, the standard treatment approach has been surgical resection. For the right-sided lesions, percutaneous mechanical thrombectomy (PMT) is a viable treatment option. Primary cardiac tumors are rare (less than $0.1 \%$ of the population) [1], and papillary fibroelastomas (PFEs) account for approximately $10 \%$ of primary cardiac tumors [2-4] and the most frequent type affecting the valves. Although they do carry the potential for embolization, they are usually discovered incidentally (via echocardiography or cardiac computed tomography/magnetic resonance imaging (CT/MRI)). Despite being traditionally detected on valvular surfaces, they do not cause valvular dysfunction. Most PFEs are located in the left heart. Here we present an unusual case of a PFE of the right atrium, allowing for PMT.

Received 07/09/2018

Review began 09/30/2018 Review ended 11/30/2018 Published 12/03/2018

(c) Copyright 2018

Bhagat et al. This is an open access

article distributed under the terms of the

Creative Commons Attribution License

CC-BY 3.0., which permits unrestricted

use, distribution, and reproduction in any medium, provided the original author and source are credited.

\section{Case Presentation}

A 72-year-old man with past medical history significant for a stroke and hypertension was undergoing routine evaluation for his muscular dystrophy. Transthoracic echocardiogram (TTE) revealed a right atrial mass measuring $1.5 \mathrm{~cm} \times 2 \mathrm{~cm}$ (Figure 1). 


\section{Cureus}

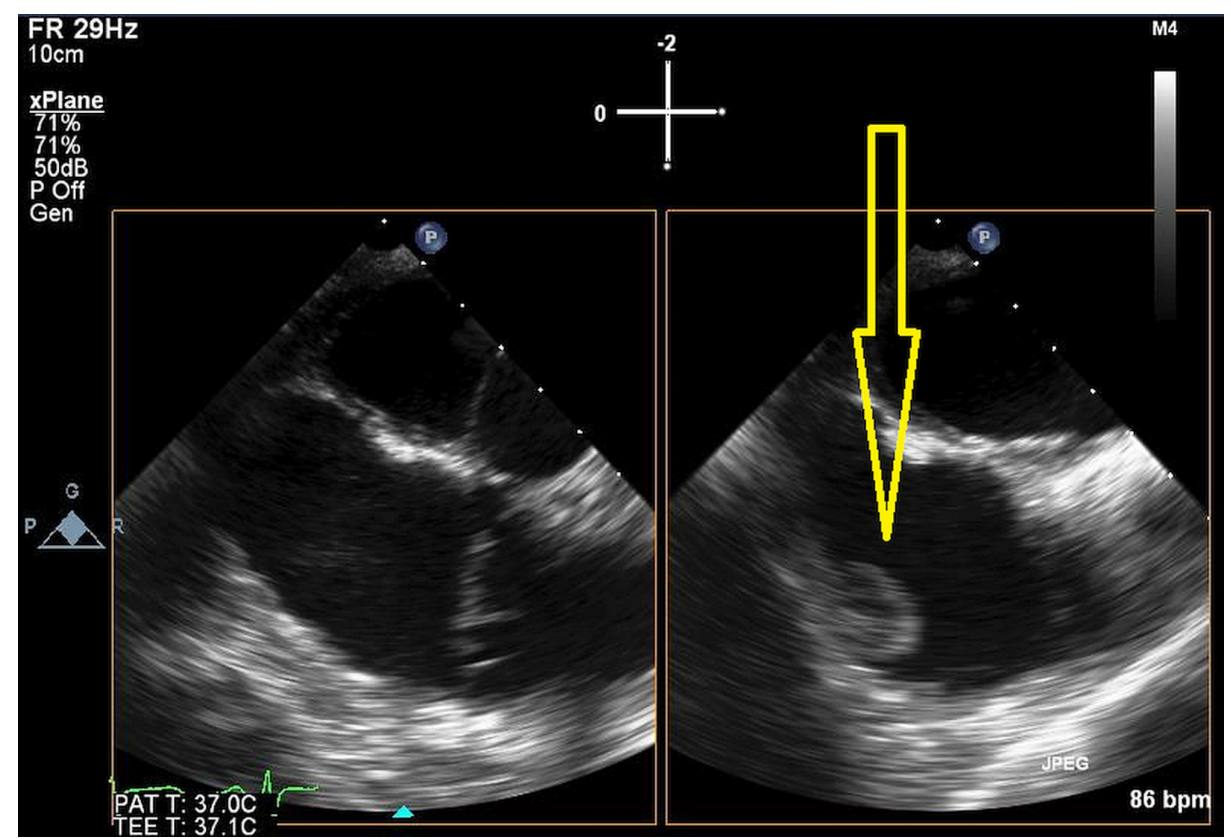

FIGURE 1: Transthoracic echocardiogram (TTE) revealed a right atrial mass measuring $1.5 \mathrm{~cm} \times 2 \mathrm{~cm}$.

Arrow as shown displays position of right heart mass.

Transesophageal echocardiogram (TEE) results were consistent with this finding (Figures 2-3).

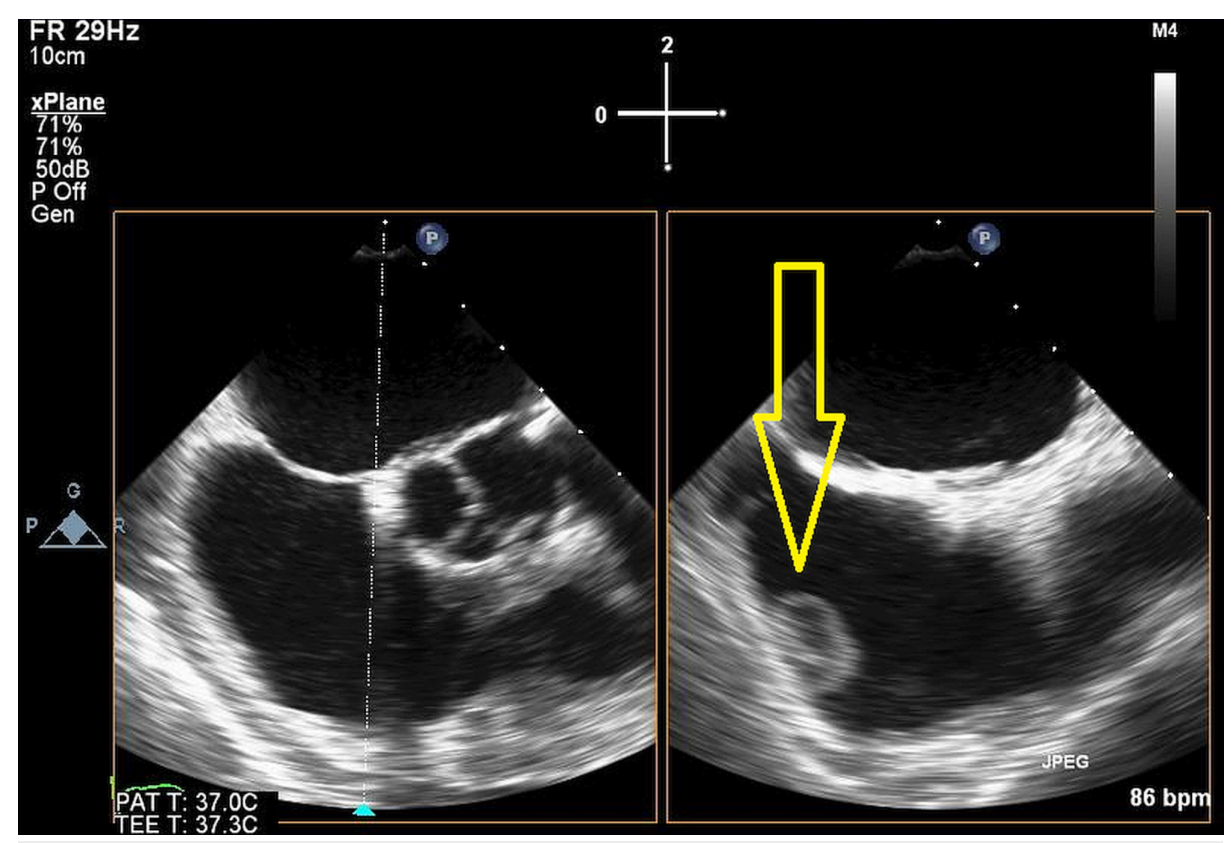

FIGURE 2: Transesophageal echocardiogram additional image.

Arrow as shown displays 2D image of right heart mass and attachment onto the surrounding wall. 


\section{Cureus}

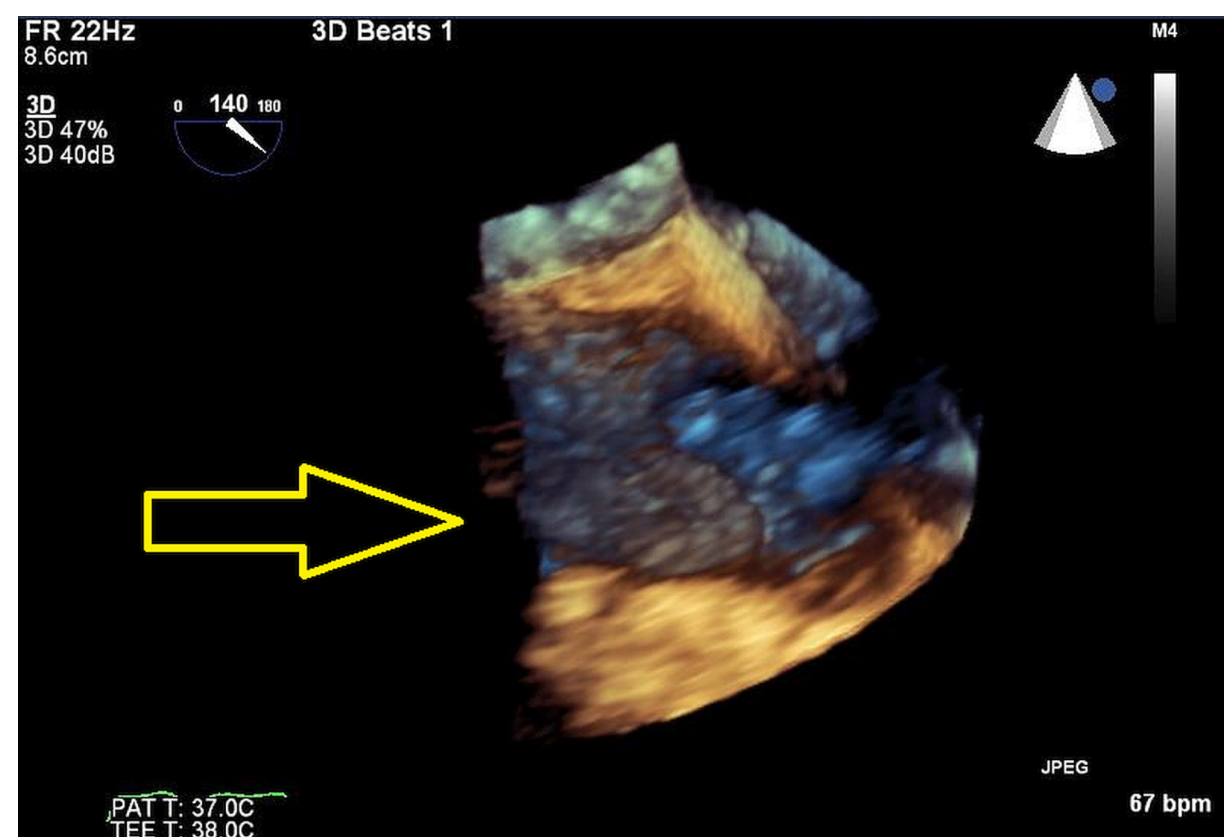

FIGURE 3: 3D model of mass.

Arrow displays position and size of mass related to the heart wall.

He was started on anticoagulation and transferred to our facility for PMT using AngioVac (Figure 4).

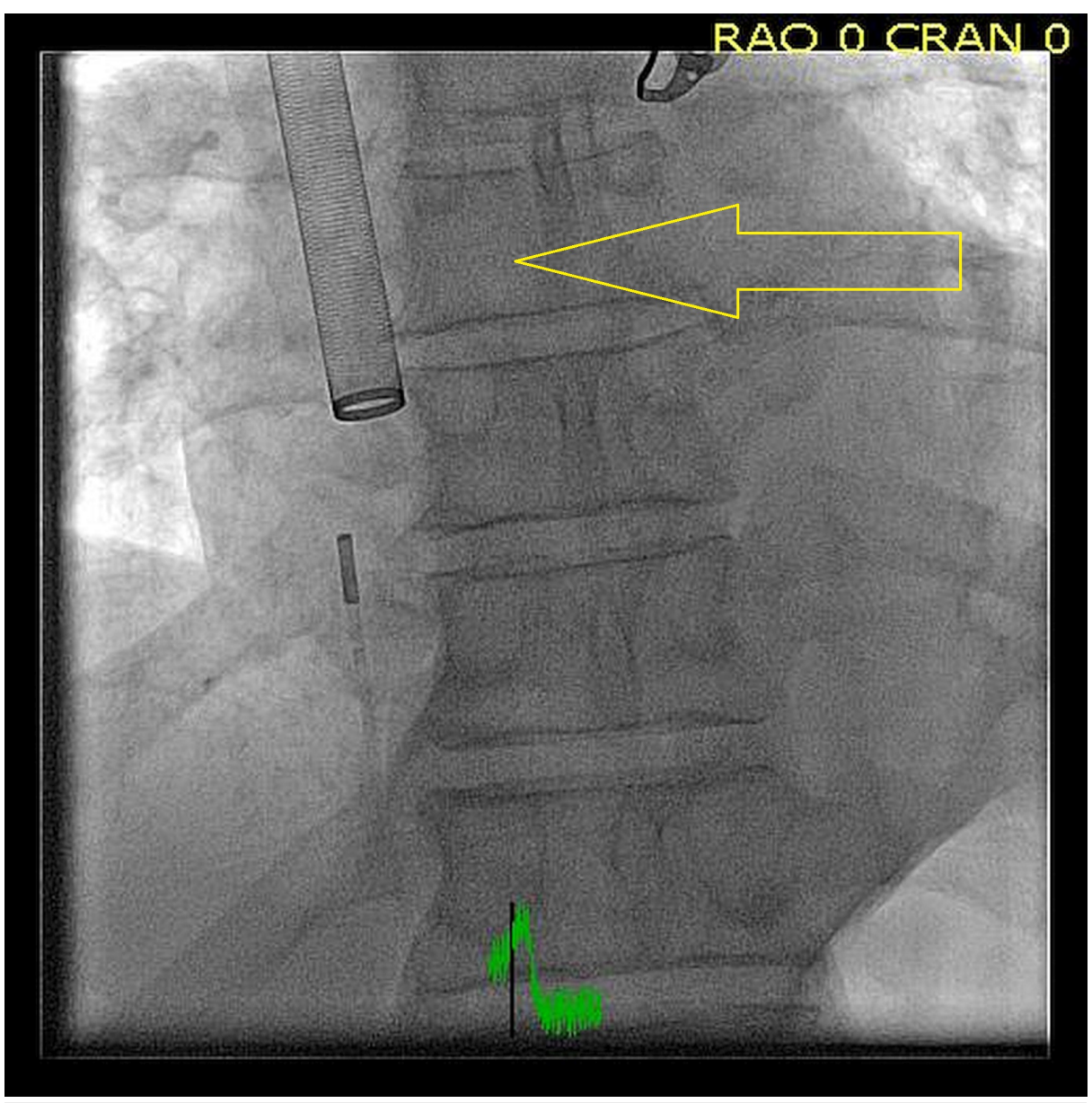

FIGURE 4: Percutaneous mechanical thrombectomy (PMT) using AngioVac. 
For this procedure, the right internal jugular vein and bilateral common femoral veins were accessed. Intracardiac echo was used. The intracardiac echo showed an approximately $1.5 \mathrm{~cm} \times 2 \mathrm{~cm}$ mass in the right atrium. This was ultimately extracted with $0 \%$ leftover tissue burden. Pump time was approximately two minutes. 22 French AngioVac cannula had been fashioned with a heat gun. This was then introduced from the right internal jugular vein. Blood was delivered via the left common femoral vein. Intracardiac echo was performed from the right common iliac vein. Tissue was sent to pathology for further review which confirmed the diagnosis of PFE. The patient was then discharged home without the need for long-term anticoagulation.

\section{Discussion}

The PMT has mostly been used as an adjunct therapy for the treatment of DVT. PMT is classified into rheolytic, rotational, or ultrasound-enhanced. Rheolytic PMT sprays a thrombolytic agent followed by pressurized saline at the thrombus [1]. The softened thrombus is then suctioned by the catheter into a collecting canister. Rotational devices function to macerate the thrombus via a high-velocity rotating helix [3]. Ultrasound-enhanced devices employ catheters containing ultrasound transducers which emit highfrequency, low-energy waves to expand and thin the fibrin component of the thrombus. This exposes plasminogen receptors and allows for higher penetration by the lytic agent. PMT, compared to surgical resection of cardiac masses, does not warrant a sternotomy or cardioplegic cardiac arrest - minimizing the associated complications [3]. The average hospitalization time after surgical resection of heart masses is approximately five days. In contrast, most patients are able to be discharged the following day with PMT. From a financial perspective, surgical resection costs approximately $\$ 130,000$, whereas PMT costs $\$ 52,000$ [1-4].

\section{Conclusions}

Upon our review of the literature, no reports were found in which PMT was applied for right-sided heart masses (tumors, vegetations, thrombi). However, PMT is successfully being used for the treatment of massive pulmonary emboli, DVTs, and ischemic strokes. In this case, the patient was started on anticoagulants after a $1.5 \mathrm{~cm} \mathrm{x} 2 \mathrm{~cm}$ right atrial mass was confirmed using TTE, TEE and intracardiac echo. The pathology report of the mass after having it totally extracted confirmed the diagnosis of PFE; therefore, the anticoagulants were discontinued. We hypothesize that PMT will lead to less morbidity and mortality, cost, and hospitalization, compared to standard surgical resection for these right-sided lesions. Further studies examining these two approaches are recommended.

\section{Additional Information \\ Disclosures}

Human subjects: Consent was obtained by all participants in this study. CAMC Institute issued approval NA. This is a case report, so IRB approval is not necessary. Conflicts of interest: In compliance with the ICMJE uniform disclosure form, all authors declare the following: Payment/services info: All authors have declared that no financial support was received from any organization for the submitted work. Financial relationships: All authors have declared that they have no financial relationships at present or within the previous three years with any organizations that might have an interest in the submitted work. Other relationships: All authors have declared that there are no other relationships or activities that could appear to have influenced the submitted work.

\section{References}

1. Reynen K: Frequency of primary tumors of the heart . Am J Cardiol. 1996, 77:107. 10.1016/S00029149(97)89149-7

2. Karthikesalingam A, Young EL, Hinchliffe RJ, Loftus IM, Thompson MM, Holt PJE: A systematic review of percutaneous mechanical thrombectomy in the treatment of deep venous thrombosis. Eur I Vasc Endovasc Surg. 2011, 41:554-565. 10.1016/j.ejvs.2011.01.010

3. Finlayson GN: Right heart thrombi: consider the cause . Can J Cardiol. 2008, 24:888.

4. Abu Saleh WK, Al Jabbari O, Ramlawi B, Reardon MJ: Cardiac papillary fibroelastoma: single-institution experience with 14 surgical patients. Tex Heart Inst J. 2016, 43:148-151. 10.14503/THIJ-14-4889 